\title{
Massive Dog Bite Injury of the Scalp in One-year Old Boy
}

\section{Marko Bašković, Anto Pajić, Zoran Barčot}

Department of Pediatric Surgery, Children's Hospital Zagreb, Zagreb, Croatia Received May 5, 2020; Accepted November 2, 2020.

Key words: Dog bite - Scalp avulsion - Child - Plastic surgery

Abstract: A one-year-old boy was referred to our Department of Pediatric Surgery with extensive scalp injury. He was bitten by a neighbour's mixed-breed dog. The wound of the forehead is primary closed while scalp is reimplanted. Due to non-acceptance on the eighth day a necrectomy of devitalized tissue was done. Before applying Integra ${ }^{\circledR}$, for 2 days, the wound was treated with a V.A.C. ${ }^{\circledR}$ system. After 14 days, Integra ${ }^{\circledR}$ was accepted and split-thickness skin graft (STSG) was transplanted from left upper leg. After 3 months the local status is satisfactory. A hair transplant is planned in the future.

Mailing Address: Marko Bašković, MD., Department of Pediatric Surgery, Children's Hospital Zagreb, Ulica Vjekoslava Klaića 16, 10000 Zagreb, Croatia; Phone: +385 136363 79; e-mail: baskovic.marko@gmail.com 


\section{Introduction}

Of all emergency pediatric conditions, dog bites account for 0.3-1.5\% (Daniels et al., 2009; Sabhaney and Goldman, 2012). Particularly at-risk group is children under 10 years of age. Lately, dog bites have been increasingly recognized as a medical and public health issue, as they leave functional, aesthetic and psychosocial consequences (Bernardo et al., 2000).

\section{Case report}

We show the case of a one-year-old boy who was referred from a general hospital to our Department of Pediatric Surgery for extensive scalp injury by a neighbour's dog (Figure 1a). In the general hospital, the wound was flushed and the child was administered ceftriaxone. A piece of scalp skin was sent in saline. Upon arrival, the child was vaccinated (tetanus-diphtheria toxoids/tetanus immune globulin). The craniogram showed no signs of fracture. At the operating table, we verified a $22 \mathrm{~cm}$ long forehead and scalp injury that extended from the left eyebrow to the middle of the scalp. A swab was taken. Immediately, thinking about the final
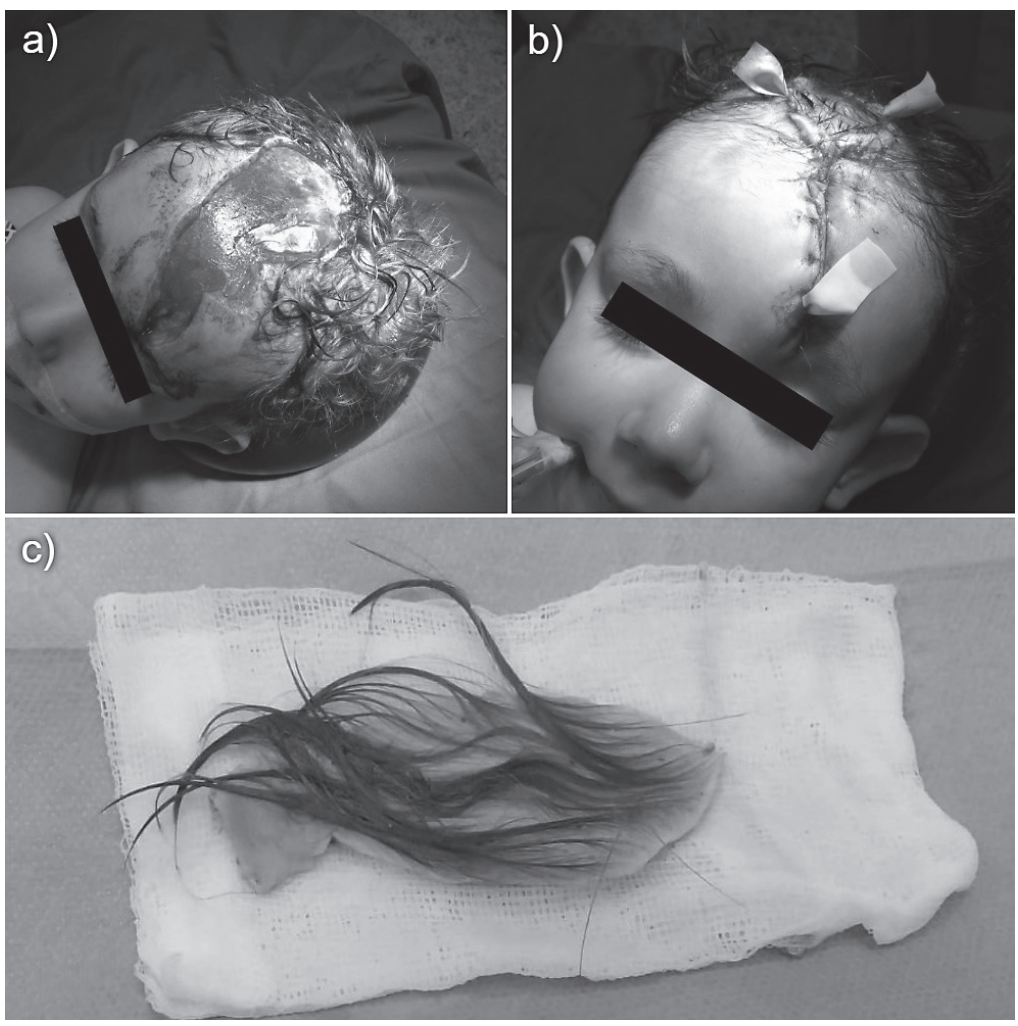

Figure 1 - a) Massive scalp injury; b) appearance after primary forehead closure and scalp reimplantation; c) scalp piece $(9 \mathrm{~cm} \times 5 \mathrm{~cm})$. 
aesthetic appearance, we decided to primary close the forehead region first. On the part of the scalp that we were unable to primarily close, we reimplanted a piece of boy's scalp measuring $9 \mathrm{~cm} \times 5 \mathrm{~cm}$. The edges of the wound were sutured with Monosyn ${ }^{\circledR} 4 / 0$ sutures. 2 drains were placed (Figure $1 b$ and c). Despite regular dressings and monitoring with appropriate antibiotic therapy (the following pathogens were isolated in the swab; Enterobacter aerogenes, Pasteurella multocida, Citrobacter freundii - all resistant to penicillin, ampicillin, amoxicillin-clavulanic acid) the reimplanted part of the scalp was not accepted (Figure 2a). On the eighth day, a necrectomy of the devitalized tissue was performed (Figure 2b). The wound edges were refreshed, treated with Microdacyn ${ }^{\circledR}$, and a V.A.C. ${ }^{\circledR}$ system $(-125 \mathrm{~mm} \mathrm{Hg})$ was set up (Figure $2 \mathrm{c}$ and d). He worked continuously for 2 days before Integra ${ }^{\circledR}$ was set up. Integra ${ }^{\circledR}$ was fenestrated before placement. After setting Integra ${ }^{\circledR} 2$ times we changed the V.A.C. ${ }^{\circledR}$ system (Figure 2e). With the acceptance of Integra ${ }^{\circledR}$, on the $14^{\text {th }}$ day a silicone layer was removed and split-thickness skin graft (STSG)

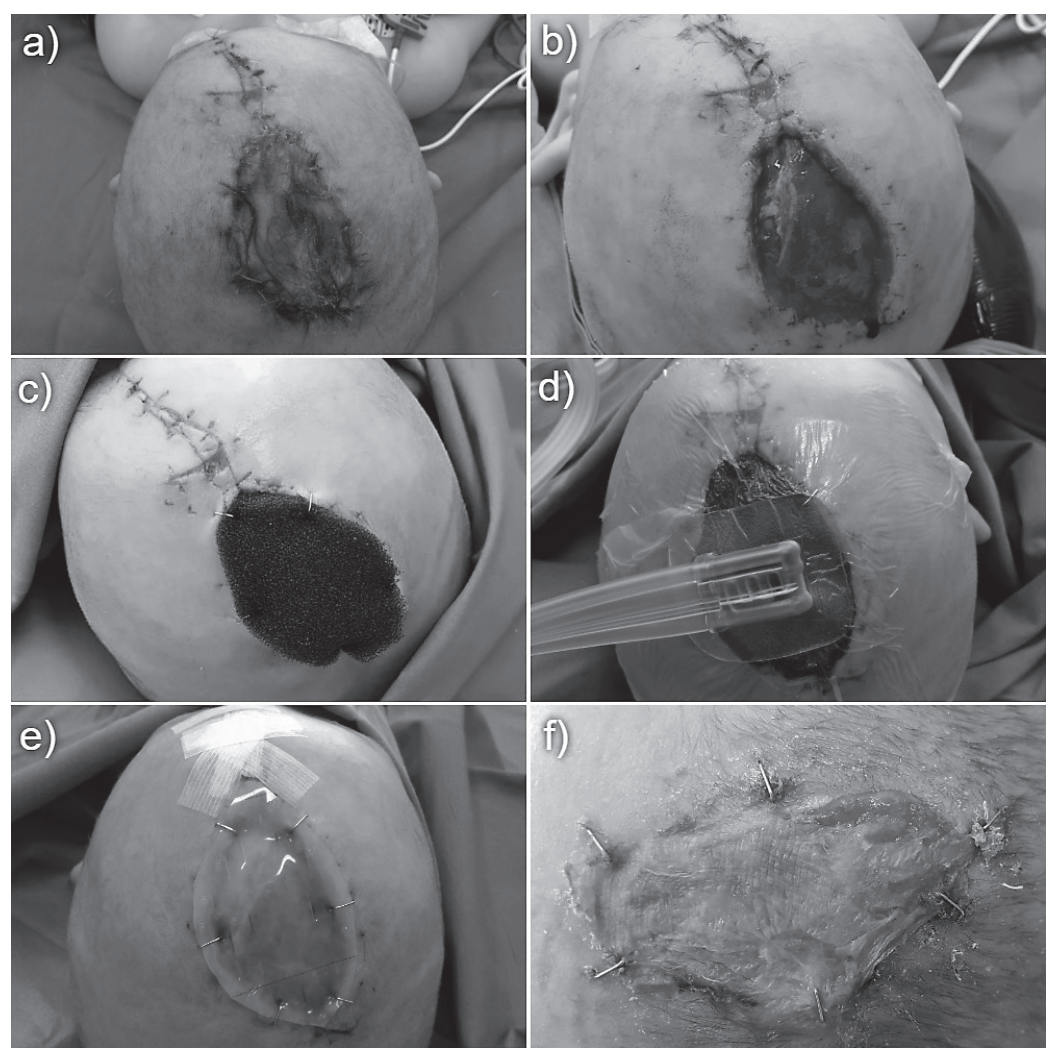

Figure 2 - a) Devitalized scalp tissue; b) refreshed wound edges after necrectomy; c) placement of a sponge; d) activated V.A.C. ${ }^{\circledR}$ system $(-125 \mathrm{~mm} \mathrm{Hg})$; e) Integra ${ }^{\circledR}$ Dermal Regeneration Template; f) accepted Integra ${ }^{\circledR}$ and split-thickness skin graft (STSG). 


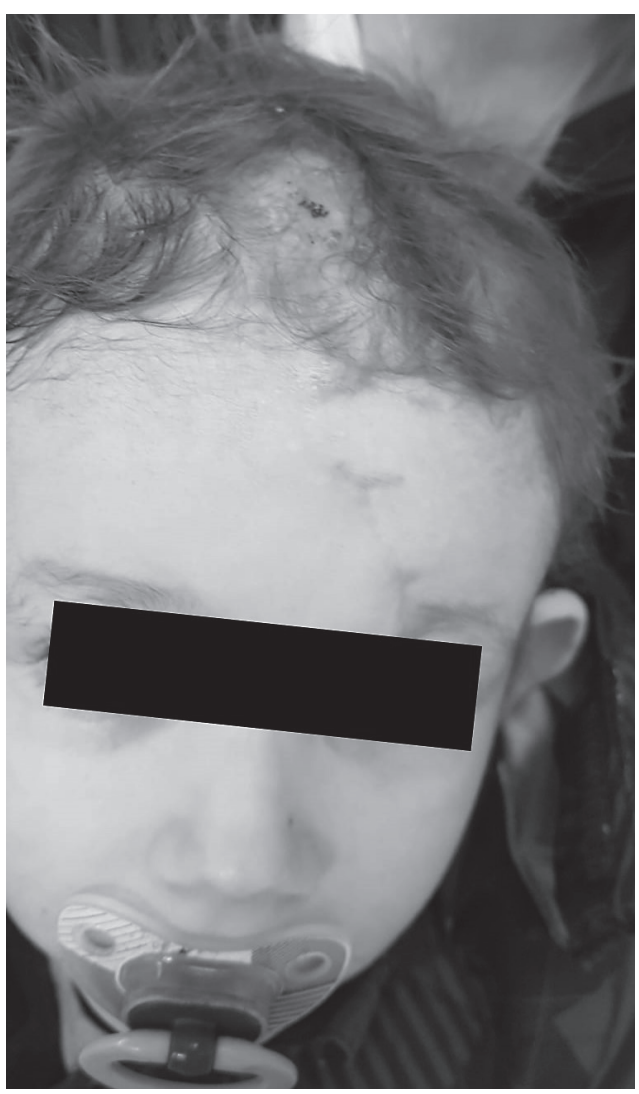

Figure 3 - Appearance of boy's forehead and scalp after 3 months.

was transplanted from the left upper leg. On the STSG Bactrigas ${ }^{\circledR}$ was placed with the V.A.C. ${ }^{\circledR}$ system. By monitoring and replacing the V.A.C. ${ }^{\circledR}$ system, STSG was accepted (Figure 2f). One month after the injury, the boy was released from the hospital. After 3 months the local status is satisfactory (Figure 3). We plan to hair transplantation in the future.

\section{Discussion}

Although in our case we continued to use the already started ceftriaxone therapy, amoxicillin-clavulanic acid and first-generation cephalosporins are used as the firstline antibiotics. Complications from dog bites include wound infection, osteomyelitis, cellulitis, septic arthritis, meningitis, sepsis, endocarditis, pneumonia and death. Rates of infection are estimated to range from $1 \%$ to $30 \%$. In addition to thorough wound treatment, tetanus vaccination is also required. According to the guidelines of the American Academy of Pediatrics, all children under the age of three must receive tetanus-diphtheria toxoids and tetanus immune globulin. For children older than three years tetanus-diphtheria toxoids is necessary if five or more years have elapsed 
since the last dose. Tetanus immune globulin is not required in children older than three years. Depending on the status of the dog, rabies prophylaxis is also important (Sabhaney and Goldman, 2012; Macedo et al., 2016).

As in our case, Bernardo et al. (2000) stated that most of the bites occur exactly from their own (27\%) or their neighbour's dog (28\%). As for the breed, Chen et al. (2013) report that most bites occur by mixed breed (23.0\%), Labrador retriever (13.7\%), Rottweiler (4.9\%), and German shepherd (4.4\%).

Due to the disproportion of the head in relation to the body and their height, younger children are more prone to injuries to the head and face (Chen et al., 2013). Subsequent, inevitable scars have aesthetic and psychological consequences for the children, especially when they found in adolescence (Daniels et al., 2009). Therefore, treatment strategies should include early psychological support for children and their families (Schalamon et al., 2006).

Satteson et al. (2015) support the option that VAC (vacuum assisted closure) provides reliable, effective, and durable dressing when traditional surgery is not an option (e.g. primary closure, healing by secondary intention, local tissue transfer, grafts, and flaps). The VAC device works by applying continuous, subatmospheric pressure to a wound through an open-cell polyurethrane sponge secured with an adherent drape. The VAC has been shown to promote wound healing through increasing blood flow and granulation tissue formation, improving oxygenation, decreasing tissue edema, and reducing bacterial load. It has also been demonstrated as a tool for assisting with the preparation of wound beds for subsequent skin grafting and for accelerating the incorporation of Integra ${ }^{\circledR}$. Satteson et al. (2015) also state, in accordance with our treatment, that standard management included VAC changes 3 times per week on open wounds, whereas the VAC was left in place for 5-7 days over skin grafts and 7-14 days over Integra ${ }^{\circledR}$.

Integra ${ }^{\circledR}$ is an artificial dermis manufactured as a synthetic bilaminate composed of a bovine collagen lattice covalently linked to chondroitin-6-sulfate and covered with a silastic epidermis. The outer layer is composed of polydimethylsiloxane and serves as an epidermal substitute providing mechanical protection, infection prevention, and moisture modulation. The inner layer promotes cellular ingrowth of fibroblasts, macrophages, and lymphocytes, allowing for the regeneration of a neovascularized tridimensional structure, known as the neo-dermis. Consistent with the study by Watts et al. (2019), we also fenestrated the Integra ${ }^{\circledR}$ matrix because fenestration permits the egress of fluids, reducing the risk of seroma or hematoma formation. Like us, they believe it to be the recommended treatment option in the medically complex patient (Watts et al., 2019).

Konofaos et al. (2014) demonstrated the successful Integra ${ }^{\circledR}$ based scalp reconstruction of a $600 \mathrm{~cm}^{2}$ defect in a two-year-old male following a dog bite injury where options for skin grafting, local flaps, and free tissue transfer were not available. Although we successfully removed the protective silicone layer after 2 weeks, Konofaos et al. (2014) did it after 3 weeks. 


\section{Conclusion}

The use of Integra ${ }^{\circledR}$, followed by an ultrathin skin graft, reduces the morbidity by eliminating the need for large regional or free tissue transfer creating a further defect at the donor site. In addition, the surgical procedure is much faster than with flaps, reducing the risk of anesthesia and the treatment-related comorbidity. Like Konofaos et al. (2014), we also believe that this technique offers another viable option for the treatment of complex scalp wounds in pediatric patients and deserves to be added to the list of reconstructive surgical techniques used by the pediatric plastic surgeons.

\section{References}

Bernardo, L. M., Gardner, M. J., O'Connor, J., Amon, N. (2000) Dog bites in children treated in a pediatric emergency department. J. Soc. Pediatr. Nurs. 5(2), 87-95.

Chen, H. H., Neumeier, A. T., Davies, B. W., Durairaj, V. D. (2013) Analysis of pediatric facial dog bites. Craniomaxillofac. Trauma Reconstr. 6(4), 225-232.

Daniels, D. M., Ritzi, R. B., O’Neil, J., Scherer, L. R. (2009) Analysis of nonfatal dog bites in children. J. Trauma 66, S17-S22 (Suppl. 3).

Konofaos, P., Kashyap, A., Wallace, R. D. (2014) Total scalp reconstruction following a dog bite in a pediatric patient. J. Craniofac. Surg. 25(4), 1362-1364.

Macedo, J. L., Rosa, S. C., Queiroz, M. N., Gomes, T. G. (2016) Reconstruction of face and scalp after dog bites in children. Rev. Col. Bras. Cir. 43(6), 452-457.

Sabhaney, V., Goldman, R. D. (2012) Child health update. Management of dog bites in children. Can. Fam. Physician 58(10), 1094-1096.

Satteson, E. S., Crantford, J. C., Wood, J., David, L. R. (2015) Outcomes of vacuum-assisted therapy in the treatment of head and neck wounds. J. Craniofac. Surg. 26(7), e599-e602.

Schalamon, J., Ainoedhofer, H., Singer, G., Petnehazy, T., Mayr, J., Kiss, K., Höllwarth, M. E. (2006) Analysis of dog bites in children who are younger than 17 years. Pediatrics 117(3), e374-e379.

Watts, V., Attie, M. D., McClure, S. (2019) Reconstruction of complex full-thickness scalp defects after dog-bite injuries using dermal regeneration template (Integra): Case report and literature review. J. Oral Maxillofac. Surg. 77(2), 338-351. 